\title{
Review of Automated Design and Optimization of MEMS
}

\author{
Achiche, Sofiane; Fan, Zhun; Bolognini, Francesca
}

Published in:

2007 IEEE International Symposium on Industrial Electronics

Link to article, DOI:

10.1109/ISIE.2007.4374941

Publication date:

2007

Document Version

Publisher's PDF, also known as Version of record

Link back to DTU Orbit

Citation (APA):

Achiche, S., Fan, Z., \& Bolognini, F. (2007). Review of Automated Design and Optimization of MEMS. In 2007 IEEE International Symposium on Industrial Electronics IEEE. https://doi.org/10.1109/ISIE.2007.4374941

\section{General rights}

Copyright and moral rights for the publications made accessible in the public portal are retained by the authors and/or other copyright owners and it is a condition of accessing publications that users recognise and abide by the legal requirements associated with these rights.

- Users may download and print one copy of any publication from the public portal for the purpose of private study or research.

- You may not further distribute the material or use it for any profit-making activity or commercial gain

- You may freely distribute the URL identifying the publication in the public portal

If you believe that this document breaches copyright please contact us providing details, and we will remove access to the work immediately and investigate your claim. 


\section{Review of Automated Design and Optimization of MEMS}

\author{
Sofiane Achiche, Zhun Fan \\ Department of Mechanical Engineering \\ Technical University of Denmark \\ Lyngby, Denmark \\ Email:sac@gst.mek.dtu.dk,zf@mek.dtu.dk
}

\author{
Francesca Bolognini \\ Department of Engineering \\ University of Cambridge \\ Cambridge, United Kingdom \\ Email: fb252@eng.cam.ac.uk
}

\begin{abstract}
In recent years MEMS saw a very rapid development. Although many advances have been reached, due to the multiphysics nature of MEMS, their design is still a difficult task carried on mainly by hand calculation. In order to help to overtake such difficulties, attempts to automate MEMS design were carried out. This paper presents a review of these techniques. The design task of MEMS is usually divided into four main stages: System Level, Device Level, Physical Level and the Process Level. The state of the art o automated MEMS design in each of these levels is investigated.
\end{abstract}

\section{INTRODUCTION}

Micro-Electro-Mechanical Systems (MEMS) are the integration of mechanical elements, sensors, actuators, and electronics on a common silicon substrate through microfabrication technology. Medical sensors, wireless communications, environments monitoring, military surveillance are some of the many applications that MEMS have been used in. MEMS technology is evolving fast, and a vast amount of conferences and scientific journals are nowadays available to keep track of current research directions, advances and results. Among the most important conferences that deal with MEMS work are the IEEE Micro Electro Mechanical Systems conference and the International Society for Optical Engineering (SPIE) conferences. Some of the most important journals are the Journal of Microelectromechanical Systems (IEEE/ASME), the Journal of Micromechanics and Microengineering (Institute of Physics), the Journal of Microlithography, Microfabrication, and Microsystems (SPIE), the Journal of Microfluidics and Nanofluidics (Springer) [1]. This review has been conducted with consideration of all the sources cited here.

The amount of research on MEMS is quite extensive but the work presented here targets only one aspect of this research: the work done on methods and techniques to automate and optimize MEMS design. The scope of this paper is limited to information available and published as of December 2006 and generally not published before 1996, unless necessary. This survey was undertaken as a prelude to a research addressing robust and automatic layout synthesis of MEMS, using evolutionary techniques, taking into account fabrication uncertainties among other constraints.

\section{Automated Design Issues}

The goals of automated design of MEMS are to shorten the development cycle of MEMS and enable the design of much more complex devices than those that can be handled today. Automated design has also the potential of sparking innovation in MEMS design, helping designers in finding solutions that go beyond their insight and experience, or helping them expand perceived design and performance limits.

MEMS hold the promise of being amenable to structured automated design due to their similarities with VLSI. However, automated design of MEMS has not seen yet a commercial use, basically because the multi-domain (mechanical, electrical, fluidics) and intrinsic 3D nature of MEMS make their design more complex. In addition, because of limitations due to fabrication technology, MEMS design is highly constrained.

This paper will present, in a compact form, a review of the work done so far in automating MEMS design. The review will also highlight points of weakness in the works developed so far and the streams of the research field that need to be explored further in order to develop more effective and supportive MEMS design tools.

To facilitate the understanding of this paper the design process of MEMS is divided into different modeling levels, as explained in the following sections.

\section{A. Modeling Levels}

In this paper the systematic modeling subdivision proposed by Senturia [2] is used as a classification of modeling issues. Such categorization will provide the stages where the design and the variety of modeling paradigms occur. Four modeling levels are proposed: System, Device, Physical and Process as shown in Figure 1 [2]. Each of them is linked by a double headed arrow symbolizing iterative exchange of information between levels, implying that the levels are not interindependent but always linked. While designing MEM devices going from top to down (system to process), is called simulation, the inverse (process to system) is called verification.

Each modeling level will be addressed and described individually. The research carried out on automated design related to each of the design levels will be reported.

These design levels for MEMS give a clear framework for understanding the review of the research work on automated design of MEMS presented in this paper. 


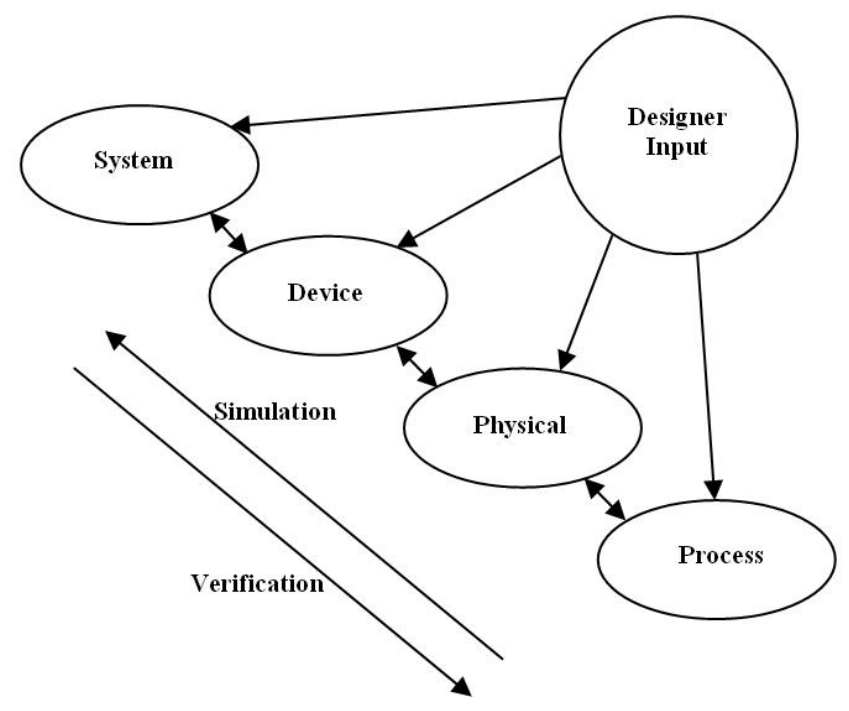

Fig. 1: Different modeling levels fro Microsystems [2]

\section{System And Device LeVel Modeling}

The system level modeling is the home of the blockdiagram descriptions and lumped-element circuit models [2]. The interactions of the MEMS component with its environment and electronics can be modeled and simulated at this level. There are two approaches to system level modeling, either using a Model-based design method or a hardware description language (HDL). In mathematical terms this relates to working with block diagrams or Ordinary Differential Equations (ODE). In the system level modeling, single MEMS devices are used. These devices are often large and complex. It is sometimes difficult to capture the physics or behavior in terms of ODEs. On the other end it is often too cumbersome to use Partial Differential Equations (PDEs) like it is done in Physical Modeling. In these cases it is possible to use Macro-Modeling, Compact Modeling or Reduced Order Modeling approaches [2]. One important prerequisite is that the extracted models must be compatible with the System level models [2, 3]. Figure 2 shows the relationships between deferential equations, meshes and models [4]. The research work on automated design of MEMS has concentrated its effort mostly on the device level of MEMS because of the high complexity of the system level.

Furthermore, some software tools also allow users to employ block diagrams or ODEs simultaneously. The MatlabSimulink environment is an example of Model-based design and Verilog-AMS, Eldo or Saber are examples of HDL-based design [3]. Due to the multi-domain and intrinsic threedimensional nature of MEMS, their design and analysis is very complex and requires access to simulation tools with finite element analysis capability. Computation cost is typically very high. A common representation that encompasses multiple energy domains is thus needed for modeling the whole system. A system-level (including MEM devices) model that reduces the number of degrees of freedom from the hundreds and thousands of degrees of freedom characterizing the meshed 3D model to as few as possible is needed [5].

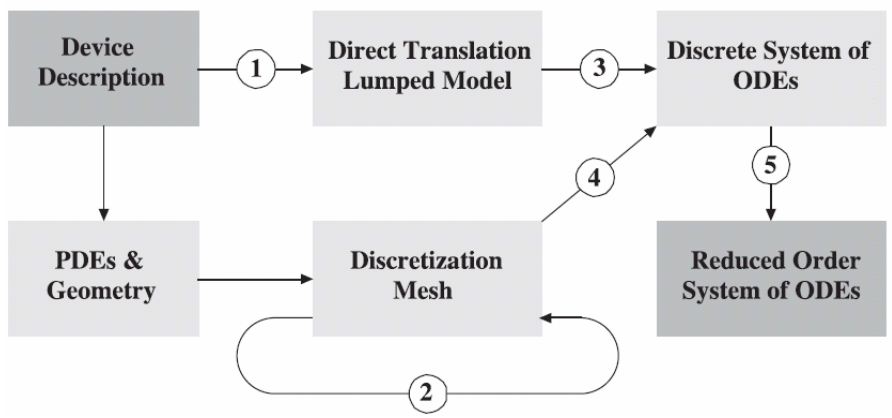

Fig. 2. Some of the routes leading from a device description to a reduced order system of ODEs. The arrows represent translations between descriptions: 1) Lumping is done by hand, either as a circuit equivalent, or as an algebraic expression; 2) adaptive meshing determines the size of the subsequent model; 3 ) circuit equivalents or algebraic expressions are turned into a suitable set of ODEs; interconnecting many of these again leads to large systems; 4) semidiscretization of the PDEs on a mesh results in a set of ODEs; 5) an algebraic model reducer takes a large system of ODEs and produces a smaller (and hence reduced order) yet equivalent system of ODEs.

Behavioral simulation on the system/device level can be accomplished using different commercial tools such as MATLAB [6, 7], Saber a multi-technology, mixed-signal simulation environment used for designing and analyzing complex mechatronic systems [8], schematic-capture IC tools like Spectre [9] and SPICE [10, 11]. Some software tools allow using geometric parameterized cell libraries like the schematicdriven design environment Architect [13] and MEMSPro [12] which is based on the popular IC layout tool, L-Edit, however the construction of behavioral models for MEMS components using these tools is completely manual which requires a specific expertise often lacking in a system designer, this issue is amplified by the complex mixed-domain concept of MEMS. This is why hand-generated models are still widely used, Electrostatic and mechanical simulations of MEMS systems have been performed by many authors and some simple handgenerated macromodels of accelerometers have been reported $[14,15]$. However, as MEMS systems become more complex, the need for models with large numbers of coupled degrees of freedom increases. Generating such models by hand is inefficient and prone to error, and can be difficult or even impossible.

In an attempt to automatically design MEMS on the system level some research works proposed different approaches that will be discussed bellow.

\section{A. Automatic Generation Of Macromodels}

The most typical way of creating macromodels is to use the lumped-element approach. Although the macromodel created by this approach is intrinsically compatible with any circuit simulators, many costly FEM/FDM simulations may be required for extracting appropriate lumped elements. Furthermore, the accuracy of the lumped-element approach is detrimentally affected if either the original device geometry is too complicated or the coupled-domain dynamical behavior is highly nonlinear. Therefore, various techniques for extracting MEMS macromodels have been proposed.

The development of a new CAD tool, AutoMM, which supports the automatic generation of dynamic macromodels for a broad class of MEMS devices such as the analog devices ADXL76 airbag accelerometer, was proposed by Swart et al. [16]. For linear or weakly nonlinear systems, the macromodels 
can be easily obtained by reducing the finite-element (FEM) or finite-difference (FDM) formulation using the Arnoldi Algorithm [17] or the quadratic techniques [18, 19, 20, 21, 22]. These approaches are very effective and accurate for linear systems with complicated geometries, but are incapable of capturing the dynamical behavior for nonlinear models. An approach used for highly nonlinear system over a wide range of operating conditions was proposed by Rewienski et al. [23, 24], where the algorithm presented has been demonstrated to be effective and accurate for highly nonlinear systems, but its performance still depends on a few parameters, which need to be adjusted more or less arbitrarily for a given application example. The reduced-order models generated by the Karhunen-Loeve/Galerkin approach [25, 26], on the other hand, are based on the basis functions extracted from an ensemble of the snapshots of the physical fields (e.g., pressure distribution or temperature distribution) under certain actuation conditions, and have been proved to be effective and accurate for macromodeling nonlinear systems too. However, this approach requires expensive coupled-domain FEM/FDM runs to provide enough snapshot data for extracting basis functions. Furthermore, the efforts required for implementing and simulating the FEM/FDM runs increase exponentially if any new physical domain is introduced into the system [27].

Other automatic extraction methods using control theoretical approaches, Krylov-subspace based approaches and commercially available reduced order modeling can be found in $[4,28,29]$ respectively.

All theses techniques deal with an existing model of the MEMS device and the automatic generation of the macromodels are used for simulation purpose. In the next subsection the automated synthesis of MEM devices geometry and characteristics will be discussed.

\section{A. Automated Synthesis of MEM Devices}

Antonsson [30] has given an overview of design synthesis research for MEMS design and different approaches were undertaken to automatically synthesize MEMS devices. Some researchers used numerical parameter optimization to optimize the geometry of a given MEMS configuration and applied it to a system level design; in Hung and Senturia [31] the method is used to capture the behavior of a pressure sensor based on the pull-in time of an electrostatically actuated microbeam, including the effects of squeeze-film damping due to ambient air under the beam. Mukherjee et al. [32] proposed a rapid layout synthesis of a lateral accelerometer from high-level functional specifications and design constraints. Component synthesis using a multi-objective genetic based evolutionary algorithm was proposed by Kamalian et al. [33, 34] and Zhou et al. $[35,36,37]$, where design solutions are synthesized from some parameterized basic MEMS building blocks using a genetic algorithm. This method has been successfully applied to generate meandering resonators and the designs obtained have been successfully fabricated. An automated approach to generate novel MEMS accelerometer configurations was proposed by Campbell [38]. A synthesis tool for the layout design of complex or arrayed MEM devices (systems that consists of a number of similar components or devices) is proposed by Juneidi [39]. In order to use such tool, the method requires that designers model topologies of MEM devices composing the arrays first. Ongkodjodjo [40] proposes an optimization and design of MEMS devices (using a microgyroscope as an example) based on a simulated annealing algorithm. The results of the simulation algorithm were verified and validated with the finite element method and the boundary element method of IntelliSuitTM [41]. A recent approach by Bolognini at al. [44] proposed a multi-criteria system-based method for MEMS, a method combining building blocks approach to their automated system models where the synthesis technique uses a Connected-Node-System Burst algorithm. The method was successfully applied to the synthesis of microresonators. All the approaches mentioned so far require prior specification of building blocks by the designers and, as an the attempt to not restrict these methods to specific types of MEM deigns, a high-level MEMS conceptual design synthesis model was proposed by Gibson [42], a case study of an optical processor manually designed using the MUMPs process is presented. Such model uses MEMS device behaviors as building blocks, which are at higher level of abstraction than that of using structural components as building blocks. However there is no elaboration, nor any new example to illustrate how the building blocks can be constructed for design synthesis. Another method of design synthesis using behavioral building blocks was also proposed by Deng at al. [43], accomplished by both forward and backward synthetic search strategies. Here again, the method was applied to existing MEMS devices but no new device concept was neither proposed nor optimized. An evolutionary approach using a genetic programming over bond graphs representation to achieve automatic design of MEMS system-level lumped elements parameter models was successfully proposed by Fan et al. [5], where an initial (starting) design is necessary to start the evolutionary algorithm. The method has been successfully applied to an automated synthesis of an RF MEM device, namely, a micro-mechanical band pass filter.

Other successful methods used to synthesize MEM devices are homogenization techniques. These methods were successfully applied to the synthesis of micro-compliant mechanisms [45][47]. A complete review of automated synthesis methods for MEM devices can be found in [46].

\section{Physical MODELING}

The Physical level of modeling is probably the best known in Mechanical engineering and MEMS engineering. It addresses the behavior of real devices in three-dimensional continuum. The governing equations are typically partial differential equations (PDE's) [2]. Various analytical models can be used to find closed-form solutions in ideal geometries, but the modeling of realistic devices usually requires either approximate solutions to the PDE's or highly meshed numerical solutions. A variety of numerical modeling tools using finite-element, boundary-element, or finite-difference methods are available for simulation at the physical level. This refers to field solvers for any physics such as solving Finite Element Models for mechanics, Navier-Stokes, or Maxwell equation just to name a few. Other discretization techniques are Boundary Element methods, Finite Volume and Volume of Flow Methods [3]. Most of macro solvers are used for automatic meshing and of 3D models of MEMS; some adaptations are made to reduce the DOF of the meshes by mixing 2D and 3D meshing as in [47]. However it is not the scope of this paper to report the various algorithms that exists for automatic meshing of 3D models. 


\section{Process Modeling}

Process simulation consists of 3D numerical simulation of process, chemistry and physics to produce accurate models after material addition and subtraction (mostly depositing and etching). Typically process simulation for MEMS is limited to the simulation of wet chemical anisotropic etching of crystal silicon and calculation of doping profiles created by implantation and diffusion.

The considerable variety of process steps in MEMS fabrication, the large number of unknown inputs, and the complexity of the calculations, render process simulation extremely time consuming or in most cases just not possible due to the lack of adequate simulation tools [2, 3]. However, some available software deals with this part of the MEMS design, such as the one-dimensional process simulator SUPREM [48] developed at Stanford University, MINIMOS [49] a software tool for the numerical simulation of field-effect transistors such as silicon bulk and SOI MOSFETs, FLOOPS and FLOODS [50] educational software developed at the university of Florida for 3-D process simulations, MICROTEC [51] an easy-to-use and Robust 2D Semiconductor Process and Device Simulator for PCs, and the Anisotropic Crystalline Etch Simulation (ACES) a PC-based 3-D etch simulator [52] (runs only on Windows 98 and NT) among others. A review on some other developed software can be found in [53].

Because of the complexity of the MEMS process, MEMS $\mathrm{CAD}$ tools favor the process emulation approach, which takes 2D masks and a description of the fabrication process to create a geometric 3D solid model. These models are built rather quickly and can not subsequently be used for physical modeling. Other recent techniques such as the "voxel-based emulation" [53] do not have this limitation and are robust to 2D-mask errors, making it possible to build highly detailed, realistic-looking virtual prototypes. A review of some of the research performed on automatic mask generation and automatic process planning is presented in the next subsection.

\section{A. Automatic Mask Generation}

An automatic method for synthesizing MEMS masklayouts is proposed by Antonsson et al. [54]. This method incorporates a forward simulation of fabrication into a general evolutionary algorithm loop, and was further developed with the work presented in $[55,56]$ by introducing robustness and some process variables in the learning algorithm. The evolutionary techniques presented were applied to simple 2D $1 / 2$ (extrusions) models. A design tool that calculates the required $2 \mathrm{D}$ mask set producing a given $3 \mathrm{D}$ model by investigating the vertical topology to the model through a trial mask set was proposed by Schmidt et al. [57, 58]. This work was based on the development introduced by Cho et al. in [59], where a new process planning technique that uses a threedimensional surface micromachined structure as input is proposed. The method decomposes an imported surface micromachined model into a set of three-dimensional models-each of which has geometry compatible with the fabrication process--, and then groups them for efficient layer generation. Finally, the fabrication order and the masks for all the layers of the structure are generated. A systematic mask synthesis method for surface micromachined MEMS was proposed by Ananthakrishnan et al. [60]. This method generates the mask automatically given a 3D geometric model of the MEMS device. The process sequence is referred to as inverse problem.
This necessitates a systematic solution of the subsequent problem, which involves automatically generating a geometric model of the MEMS device given the masks. A systematic and implementation-independent framework for the geometric modeling of MEMS is presented in order to solve the forward and inverse problems for general surface-micromachined devices.

\section{CONCLUSION}

The paper summarizes the current status of automated design of MEMS. The situation is that in many areas it is still in very early stages. Most presented works deal with only one level of automated design apart from very few.

A relatively mature part of design automation is the model reduction techniques, where very good results were obtained to help designers to avoid the use of FEAs to simulate MEMS behavior in all design stages.

The system-level modeling, although giving good results mostly using artificial intelligence techniques, was mainly applied to very simple MEMS devices and/or very simple combination of MEMS components. Applications on more complex devices are needed to validate the level of accuracy and robustness of such techniques.

On the physical level, the existing software tools are sufficiently developed. However, when it comes to the process level, very few works approached the task successfully. Moreover, they all only address simple geometries and few process variables. A more standard way of MEMS processing is the line to follow in order to make the models follow the constraints induced by the process, since there is no point in designing MEMS if it is impossible or too costly to produce /fabricate them.

This work highlighted how automated design of MEMS is clearly divided into modeling levels and the lack of link between these levels in the various techniques used for automated design. It is clear that there is a need for automated design of MEMS to focus more on the strong link that exists between design and manufacturing of the proposed models. For example, design robustness of the automatically generated MEMS designs, should be further investigated so that the resulting designs are less sensitive to fabrication errors and inherent geometric uncertainties. An evolutionary approach for robust design of MEMS was proposed by Fan et al. in [61], where a robust design method for layout synthesis of MEM resonators is formulated as a multi-objective constrained optimization problem with certain assumptions and treated by a special constrained genetic algorithm. Case studies based on layout synthesis of a crab-leg resonator and a comb-driven micro-resonator show that the approach proposed can lead to design results that meet the target performance and are less sensitive to geometric uncertainties than typical designs.

\section{ACKNOWLEDGMENTS}

Financial support from the Natural Sciences and Engineering Research Council of Canada (NSERC) under Post Doctoral grant BP-328508-2006 is gratefully acknowledged. The Technical University of Denmark is gratefully acknowledged for hosting Sofiane Achiche as a researcher. 


\section{REFERENCES}

1. White, A., "A Review of Some Current Research in Micro-ElectroMechanical Systems (MEMS) with Defense Applications", Scientific and Technical Reports, Government of Australia, Department of Defense, 2002, DSTO-GD-0316.

2. Senturia, S.D., Microsystem Design. Kluwer Academic Publishers 2001. ISBN-0-7923-7246-8.

3. Van Kuijk, J., Schropfer, S.D. and DaSilva, M., "Design Automation for MEMS/MST", Design, Automation and Test in Europe Conference \& Exhibition, Munich, Germany, 2005.

4. Rudnyi, E.B., Korvink, J.G., "Review: Automatic Model Reduction for Transient Simulation of MEMS-based Devices", Sensors Update vol. 11, p. 3-33, 2002.

5. Fan, Z., Seo, K., Hu, J., Rosenberg, R. C., Goodman, E. D. , "System-Level Synthesis of MEMS via Genetic Programming and Bond Graphs", Proceedings of the 2003 Genetic and Evolutionary Computation Conference, pp.2058-2071, 2003.

6. MathWorks Web Page, http://www.matlab.com/, TheMathWorks, Inc., 24 Prime Park Way, Natick, MA 01760-1500.

7. Fedder, G. K., "Simulation of Microelectromechanical Systems", Ph.D. Thesis, Department of Electrical Engineering and Computer Science, University of California at Berkeley, 1994.

8. Getreu, I., "Behavioral Modelling of Analog Blocks using the SABER Simulator", Proceedings on Microwave Circuits and Systems, pp 977-980, August 1989.

9. Cadence Web Page, http://www.cadence.com, Cadence Design Systems, Inc., 555 River Oaks Parkway, San Jose, CA 95134.

10. Nagel, L. W., "SPICE2: A Computer Program to Simulate Semiconductor Circuits," ERL-M520, University of California, Berkeley, 1975.

11. Fedder, G. K. and Howe, R. T., "Multimode Digital Control of a Suspended Polysilicon Microstructure", Journal of Microelectromechanical Systems, pp 283-297, vol 5, No. 4, 1996.

12. SoftMEMS Web Page, http://www.softmems.com/, SoftMEMS, 14109 Capri Dr, Los Gatos, CA 95032.

13. Coventor Web Page, http://www.coventor.com/, Coventor, Inc., 4000 CentreGreen Way, Suite 190, Cary, NC 27513.

14. Bart, S.F. and Samuels, H.R., "Design Techniques for Minimizing Manufacturing Variations in Surface Micromachined Accelerometers", ASME WAM, DSC, vol. 59, pp. 427-433, 1996.

15. Bart, S.F., Proceedings of the 5th ACR 'S GD Physic: Design Workshop, pp. 71-75, 1996.

16. Swart, N. R., Bart, S.F., Zaman, M.H., Mariappan, M., Gilbert, J.R., and Murphy, D., "AutoMM: Automatic Generation of Dynamic Macromodels for MEMS Devices", IEEE Proceedings of the Eleventh Annual International Workshop on Microelectromechanicalsystems, 1998. pp. 178-183.

17. Silveira, L. M. Kamon, M., Elfadel, I. and White, J., "A CoordinateTransformed Arnoldi Algorithm for Generating Guaranteed Stable Reduced-Order Models of RLC Circuits", Computer Methods in Applied Mechanics and Engineering, vol. 169, no 3-4 (19 ref.), pp. 377-389, 1999.

18. Bechtold T., Rudnyi, E.B. and Korvink, J.G., "Automatic Generation of Compact Electro-thermal Models for Semiconductor Devices", IEICE Transactions on Electronics, vol. 86, pp. 459-65, 2003.

19. Chen Y and White J, "A Quadratic Method for Nonlinear Model Order Reduction”, Proceedings of Modeling and Simulation of Microsystems, pp. 477-80, 2000.

20. Wang, F., and White, "Automatic model order reduction of a microdevice using the Arnoldi approach", ASME IMECE'98 DSC, vol 66 pp 527-30, 1998.

21. Yang Y.J. and Yu, C.C., "Extraction of heat-transfer macromodels for MEMS devices", Journal of Micromechanics and Microengineering, vol. 14, No 4, pp. 587-596, 2004.
22. Yang, Y-J., Kamon, M., Rabinovich, V.L., Ghaddar, C., Deshpande, M., Greiner, K., and Gilbert, J.R., "Modeling Gas Damping and Spring Phenomena in MEMS with Frequency Dependent Macro-Models", Proceedingds of the 14th IEEE International Conference on MEMS, pp 365-368, 2001.

23. Rewienski, M., and White, J., "A Trajectory Piecewise-Linear Approach to Model Order Reduction and Fast Simulation of Nonlinear Circuit and Micromachined Devices", Proceedings of Computer-Aided Design, pp. 252-257, 2001.

24. Rewienski, M., and White, J., "Improving Trajectory Piecewise-Linear Approach to Nonlinear Model Order Reduction for Micromachined Devices Using an Aggregated Projection Basis", Proceedings of Modeling and Simulation of Microsystems, pp. 128-131, 2002.

25. Hung, E., Yang, Y-J., and Senturia, S.D., "Low-Order Models for Fast Dynamical Simulation of MEMS Microstructures", Proceedings of Transducers'97, pp. 1101-1104, 1997.

26. Park, H. M. and Lee, M.W., "An Efficient Method Of Solving The Navier-Stokes Equations For Flow Control”, International Journal for Numerical Methods in Engineering, vol. 41, No 6, pp. 1133-1151, 1998.

27. Yang, Y.J., Cheng, S.Y. and Shen, K.Y., "Macromodeling of CoupledDomain MEMS Devices with Electrostatic and Electro Thermal Effects", Journal of Micromechanics and Microengineering, vol. 14, No8, pp. 1190-1196, 2004.

28. Antoulas, A.C., Approximation of Linear Dynamical Systems, Wiley Encyclopedia of Electrical and Electronics Engineering, (Ed.: J.G. Webster), 11, pp. 403-422, 1999.

29. Ostergaard, D. and Gyimesi, M., "Finite Element Based Reduced Order Modeling of Micro Electro Mechanical Systems (MEMS)", Proceedings of the 3rd International Conference on Modeling and Simulation of Microsystems, pp. 684-687, 2000.

30. Antonsson, E. K., Microsystem Design Synthesis, Chapter 5 of Formal Engineering Design Synthesis, Antonsson, E. K. \& Cagan, J., (eds), Cambridge University Press, pp. 126-169, 2001.

31. Hung, E., and Senturia, S.D., "Generating Efficient Dynamical Models for Microelectromechanical Systems from a Few Finite-Element Simulation Runs", Journal of Microelectromechanical Systems, vol. 8, no3, pp. 280289, 1999.

32. Mukherjee, T., Zhou, Y., Fedder, G. K., "Automated Optimal Synthesis of Microaccelerometers", Technical Digest of the 12th IEEE International Conference on Micro Electro Mechanical Systems (MEMS '99), pp. 326$331,1999$.

33. Kamalian, R., "Evolutionary Synthesis of MEMS Devices," Doctoral Dissertation, Department of Mechanical Engineering, University of California at Berkeley, 2004.

34. Kamalian, R., Agogino, A.M., and Takagi, H., "The Role of Constraints and Human Interaction in Evolving MEMS Designs: Microresonator Case Study", Proceedings of DETC/DAC, 2004 Design Engineering Technical Conference, Design Automation Track, CD ROM, ISBN \# I710CD, 2004.

35. Zhou, N., "Simulation and Synthesis of MicroElectronicMechanical Systems" PhD Thesis, UC Berkeley, Berkeley, CA, 8, 2002.

36. Zhou, N., Agogino, A. M., and Pister, K. S., “Automated Design Synthesis for Micro-Electro-Mechanical Systems (MEMS)”, CD-ROM Proceedings of ASME Design Automation Conference, 2002.

37. Zhou, N., Zhu, B., Agogino, A. M. and Pister, K. S. J., "Evolutionary Synthesis of MEMS MicroElectronicMechanical Systems Design", Proceedings of the Artificial Neural Networks in Engineering (ANNIE2001), 11, ASME Press, pp.197-202, 2001.

38. Campbell, M. I., "An Automated Approach to Generating Novel MEMS Accelerometer Configurations," Texas-Area MEMS Workshop, Oral presentation, 2001.

39. Juneidi, Z., Torki, K., Charlot, B., and Courtois, B., "MEMS Synthesis and Optimization," In: Design, Test, Integration, and Packaging of MEMS/MOEMS, Coutois, B. etal. (eds), SPIE, 4408, pp. 159-164, 2001.

40. Ongkodjodjo, A. and Tay, F.E.H., "Global Optimization and Design for Microelectromechanical Systems Devices Based on Simulated Annealing", Journal of Micromechanics and Microengineering, pp. 878-897, 2002. 
41. http://www.intellisensesoftware.com/

42. Gibson, D., Purdy, C., Hare, A., and Beyette F. Jr., "Design Automation of MEMS Systems Using Behavioral Modeling", Proceedings of the IEEE Great Lakes Symposium on VLSI, pp. 266-269, 1999.

43. Deng, Y.M. and Lu, W.F., "A Conceptual Design Synthesis Framework for Micro-Electro-Mechanical Systems (MEMS)", In Proceedings of the ASME Design Engineering Technical Conference, \#DETC2004-57235, 2004.

44. Bolognini, F., Shea, K, Seshia, A., Vale, C.W., "A Multicriteria System Based Method for Simulation-Driven Design Synthesis", ASME 2006 International Design Engineering Technical Conferences, 2006.

45. Bensdøe M.P. and Sigmund O., Topology Optimisation: Theory, Methods and Applications, Springer, NY, 2003.

46. Ananthasuresh, S.G.K., Optimal Synthesis Methods for MEMS (Microsystems), Springer; 1 edition, 2003, ISBN 1402076207.

47. Su, Y. and Chong, C. S., "Automatic Mixed-dimensional MEMS Modeling", Proceedings of the IEEE/ASME International Conference on Advanced Intelligent Mechatronics, pp. 243-248, 2005.

48. Hansen, S., and Deal, M., "SUPREM-IV.GS. Two Dimensional Process Simulation for Silicon and Gallium, Arsenide", Integrated Circuits Laboratory, Stanford University, Stanford, California 94305, http://wwwtcad.stanford.edu/tcad/programs/suprem-IV.GS/Book.html, 1993.

49. http://www.iue.tuwien.ac.at/

50. Law, M., FLOODS/FLOOPS Introduction. http://www.tec.ufl.edu/ flooxs/, 2002 .

51. http://www.siborg.ca/

52. http://mass.micro.uiuc.edu/research/completed/aces/pages/home.html

53. Tushar, U., "Accurate and Robust Geometric Modeling for Simulation Of IC and MEMS Fabrication Processes", Proceedings of the 2005 ACM Symposium on Solid and Physical Modeling, pp. 257 - 266, 2005.

54. Li, H. and Antonsson, E.K., "Evolutionary Techniques in MEMS Synthesis”, ASME Design Engineering Technical Conferences, 1998.

55. Ma, L. and Antonsson, E. K., "Mask-Layout and Process Synthesis for MEMS", MSM2000, Modeling and Simulation of Microsystems, Semiconductors, Sensors and Actuators, IEEE, 2000.

56. Ma, L. and Antonsson, E. K., "Applying Genetic Algorithms to MEMS Synthesis", ASME International Mechanical Engineering Congress and Exposition, ASME, 2000.

57. Schiek, R. L. and Schmidt, R.C, "Automated Surface Micro-Machining Mask Creation from a 3D Model", Microsystem Technologies, vol. 12, n 3, pp. 204-207, 2006.

58. Schiek, R. L. and Schmidt, R.C., "Automated and Integrated Mask Generation from a CAD Constructed 3D Model", Technical Proceedings of the Nanotechnology Conference and Trade Show - NSTI Nanotech, pp. 443-446, 2005.

59. Cho, S., Lee, K. and Kim, T., "Development of a Geometry-Based Process Planning for Surface Micromachining", International Journal of Production Research, vol.40, No. 5, pp. 1275-1293, 2002.

60. Ananthakrishnan, V., Sarma, R. and Ananthasuresh, G.K., "Systematic Mask Synthesis for Surface Micromachined Microelectromechanical Systems", Journal of Micromechanics and Microengineering, vol. 13, pp. 927-941, 2003.

61. Fan, Z., Wang, J., Wen, M., Achiche, S., Goodman, E and Rosenberg, R, "An Evolutionary Approach For Robust Layout Synthesis of MEMS", accepted as a book chapter in Evolutionary Computation in Dynamic and Uncertain Environment, Springer, 26 pages, 2007. 